\title{
Urgences
}

\section{Identités (minuscules)}

Numéro 16, mars 1987

D.G. Jones : d'un texte, d'autres

URI : https://id.erudit.org/iderudit/025407ar

DOI : https://doi.org/10.7202/025407ar

Aller au sommaire du numéro

Éditeur(s)

Urgences

ISSN

0226-9554 (imprimé)

1927-3924 (numérique)

Découvrir la revue

Citer ce document

(1987). Identités (minuscules). Urgences, (16), 88-93.

https://doi.org/10.7202/025407ar

Ce document est protégé par la loi sur le droit d'auteur. L'utilisation des services d'Érudit (y compris la reproduction) est assujettie à sa politique d'utilisation que vous pouvez consulter en ligne.

https://apropos.erudit.org/fr/usagers/politique-dutilisation/
Cet article est diffusé et préservé par Érudit.

Érudit est un consortium interuniversitaire sans but lucratif composé de l'Université de Montréal, l'Université Laval et l'Université du Québec à Montréal. Il a pour mission la promotion et la valorisation de la recherche. https://www.erudit.org/fr/ 


\section{Identités (minuscules) \\ Diane Ally}

Née à Drummondville en 1957. Membre du comité de direction de la revue Ellipse. Prépare actuellement une maîtrise en littérature canadienne comparée à l'Université de Sherbrooke.

\section{Claude Beausoleil}

Né à Montréal en 1948. Poète et critique. A publié vingt-cinq livres dont Au milieu du corps l'attraction s'insinue (Ed. du Noroît, 1980 - prix Emile-Nelligan) et Il y a des nuits que nous habitons tous (id. et Le Castor astral (France), 1986). A signé l'introduction ("Les langues du territoire») de l'anthologie Dix poètes anglophones du Québec. Enseigne au Collège Edouard-Montpetit.

\section{Marie Bélisle}

Née à Jonquière en 1958. A publié Noces suivi de L'itinéraire désirant (Ed. du Noroît, 1983) et Nous passions (id., 1986). Termine actuellement une maîtrise en études littéraires à l'Université du Québec à Rimouski.

\section{Florence Bernard}

Née à Argenteuil (France) en 1960. Étudie actuellement à la maîtrise en littérature canadienne comparée à l'Université de Sherbrooke.

\section{Jean-Antonin Billard}

Né à Tours (France) en 1930. Au Québec depuis 1953. Critique et traducteur. Membre de l'Association canadienne des traducteurs littêraires. Enseigne le cinéma au Collège Edouard-Montpetit.

\section{Michael Bishop}

Nê à Londres (Angleterre) en 1938. Critique. A publié, entre autres, The Contemporary Poetry of France (Ed. Rodopi (Amsterdam), 1985) et, en collaboration, The Language of Poetry: Crisis and Solution (id., 1980). Directeur de Dalhousie French Studies. Enseigne à Dalhousie University (Halifax).

\section{Louise Blouin}

Née à Montréal en 1949. Spécialisée en recherche sur les textes de radio et de télévision québécois, elle a participé à plusieurs livres sur le sujet et amassé d'importantes archives. Directrice de production aux Ecrits des Forges. Chargée de cours à l'Université du Québec à Trois-Rivières.

\section{Joseph Bonenfant}

Né à Saint-Narcisse de Champlain en 1934. Poète, romancier, critique et essayiste. A publié, entre autres, Grandes aires (Ecrits des Forges, 1984) et, en collaboration, Entre nous la neige (id., 1986). Cofondateur et codirecteur de la revue Ellipse. Enseigne à l'Université de Sherbrooke.

\section{Raymond Chamberlain}

Né à Savannah (Georgie, Etats-Unis) en 1943. Traducteur. A traduit, entre autres, L'amélanchier de Jacques Ferron (The Juneberry Tree, Harvest House Publ., 1975) et Jos Connaissant de Victor-Lévy Beaulieu (même titre, Exile Ed., 1982 - prix de traduction du Conseil des arts). Enseigne la philosophie au Collège Vanier. 


\section{Cécile Cloutier}

Née à Québec en 1930. Poète. A publié huit recueils dont les sept premiers sont maintenant réunis dans L'écouté (1960-1983) (l'Hexagone, 1986). Dirige un Centre de recherches en poésie québécoise d'aujourd'hui à l'Université de Toronto. Présidente de la Société canadienne d'esthétique. Enseigne à l'Université de Toronto.

\section{Hugues Corriveau}

Né à Sorel en 1948. Poète, romancier, critique et essayiste. A publié une dizaine de livres dont Mobiles (Ed. Les Herbes rouges, 1987) et, en collaboration, A double sens. Echanges sur quelques pratiques modernes (id, 1987). Termine un doctorat en création à l'Université de Sherbrooke. Actuellement secrétaire de rédaction (série «Auteur/e) à la NBJ. Enseigne au Collège de Sherbrooke.

\section{Louise Cotnoir}

Née à Sorel en 1948. Poète et critique. A publié, entre autres, Plusieures (Écrits des Forges, 1984) et Les rendez-vous par correspondance suivi de Les prénoms (Ed. du Remue-ménage, 1984). Enseigne au Collège de la Région de l'amiante.

\section{Jean-Paul Daoust}

Né à Valleyfield en 1946. Poète et romancier. A publié une dizaine de livres dont Dimanche après-midi (Écrits des Forges, 1985) et Les garçons magiques (VLB, 1986). Actuellement au comité de rédaction de la revue Estuaire. Enseigne au Collège Edouard-Montpetit.

\section{Normand de Bellefeuille}

Né à Montréal en 1949. Poète, critique et essayiste. A publié une vingtaine de livres dont Le livre du devoir (Ed. Les Herbes rouges, 1983 - prix Emile-Nelligan) et Catégoriques un deux et trois (Ecrits des Forges, 1986 - grand prix de poésie de la Fondation Les Forges). Actuellement secrétaire de rédaction (série "Première ligne») à la NBJ. Enseigne au Collège de Maisonneuve.

\section{Robert Dehin}

Né en Belgique en 1952. A Montréal depuis 1965. Etudie actuellement en traduction à l'Université Concordia.

\section{Michael Delisle}

Né à Longueuil en 1959. Poète. A publié, entre autres, Mélancolie (Ed. NBJ, 1985) et Les changeurs de signes (NBJ, no 192, 1987; recueil dans lequel est d'ailleurs repris Mélancolie).

\section{Monique Dumais}

Née à Rimouski en 1939. Théologienne, chercheuse en éthique. A publié, entre autres, La mère dans la société québécoise. Étude éthique d'un modèle à partir de deux journaux féministes: «La Bonne parole» (1913-1958) et «Les Têtes de pioche» (1976-1979) (Les documents de l'ICRAF, no 5, 1983). Enseigne à l'Université du Québec à Rimouski.

\section{Louise Dupré}

Née à Sherbrooke en 1949. Poète et critique. A publié, entre autres, La peau familière (Ed. du Remue-ménage, 1983 - prix Alfred-DesRochers) et, en collaboration, «Quand on a une langue, on peut aller à Rome» (NBJ, no 188, 1986). Termine 
actuellement un doctorat sur la nouvelle écriture québécoise au féminin à l'Université de Montréal. Enseigne au Collège de la Région de l'amiante.

\section{Aline Elie}

Née à Sainte-Elisabeth de Warwick en 1946. A étudié la traduction à l'Université de Montréal. Actuellement responsable de la traduction et assistante au département de français langue seconde à la Commission scolaire Eastern Townships.

\section{Célyne Fortin}

Née à La Sarre en 1943. Poète et artiste. A publié, entre autres, Femme fragmentée (Ed. du Noroît, 1982) et Au coeur de l'instant (id., 1986). Cofondatrice et codirectrice des Ed. du Noroît.

\section{Camille Fournier}

Née à Granby en 1946. Présente comme mémoire de maitrise, à l'Université de Montréal, la traduction de The Sun is Axeman (Le soleil cogne), troisième recueil de D.G. Jones. Étudie actuellement en linguistique à l'Université du Québec à Montréal. Travaille à la commercialisation des bases de données de l'Office de la langue française, à Montréal.

\section{Yvette Francoli}

Née à Alger (Algérie). Coauteure, avec Antoine Sirois, de l'édition critique d'Un homme et son péché de Claude-Henri Grignon (PUM, 1986). Achève actuellement un doctorat sur les écrits de Salvador Dali à l'Université de Sherbrooke. Chargée de cours à la même université.

\section{Madeleine Gagnon}

Née à Amqui en 1938. Poète, romancière et essayiste. A publié une quinzaine de livres dont Autographie (rétrospective 1968-1979) (VLB, 1982) et Les fleurs du catalpa (id., 1986 - prix du Journal de Montréal, section poésie). A enseigné à l'Université du Québec à Montréal de 1968 à 1982.

\section{Michel Gay}

Né à Montréal en 1949. Poète. A publié, entre autres, Eclaboussures (VLB, 1982) et Mentalité, détail (NBJ, no 174, 1986). Cofondateur et codirecteur de la NBJ, qu'il quitte en 1986. Directeur général de l'Union des écrivains québécois.

\section{André Gervais}

Né à Montréal en 1947. Poète et essayiste. A publié, entre autres, Du muscle astérisque (NBJ, no 180, 1986) et La raie alitée d'effets. Apropos of Marcel Duchamp (HMH, 1984). Enseigne à l'Université du Québec à Rimouski.

\section{Richard Giguère}

Né à Lac Mégantic en 1943. Critique et essayiste. A publié, entre autres, Exil, révolte et dissidence (PUL, 1984), sur la poésie québécoise et canadienne comparée de 1925 à 1955, et, en collaboration, A l'ombre de DesRochers (La Tribune/Ed. de l'Université de Sherbrooke, 1985). Enseigne à l'Université de Sherbrooke.

\section{Robert Giroux}

Né à Montréal en 1944. Poète, critique et essayiste. A publié, entre autres, Du fond redoutê (Triptyque, 1986) et, en collaboration, Les aires de la chanson québé- 
coise (id., 1984). Dirige la revue Moebius et les Ed. Triptyque. Enseigne à l'Université de Sherbrooke.

\section{Patricia Godbout}

Née à Verdun en 1955. Membre agréée de la Société des traducteurs du Québec et codirectrice de la revue Ellipse. Rédige actuellement un mémoire de maîtrise en littérature canadienne comparée à l'Université de Sherbrooke.

\section{Yves Gosselin}

Né à Sherbrooke en 1959. Poète.

\section{Monique Grandmangin}

Née à Témiscaming en 1941. Collaboratrice régulière à la revue Ellipse dont elle fait partie du comité de rédaction depuis 1970. Chargée de cours de traduction à l'Université de Sherbrooke.

\section{Gilles Hénault}

Né à Saint-Majoric en 1920. Poète, critique et traducteur. A publié, entre autres, Signaux pour les voyants (rétrospective 1941-1962) (l'Hexagone, 1972) et $\ddot{\mathrm{A}}$ l'inconnue nue (Parti pris, 1984).

\section{Denise Houle}

Née à Montréal en 1935. Auteur de livres pour enfants, dont Lune de neige (La Société de Belles-Lettres, 1977 - prix de littérature de jeunesse du Conseil des arts). Étudie actuellement en traduction à l'Université de Montréal. Technicienne en information à l'Office de la langue française, à Montréal.

\section{Gilbert Langevin}

Né à La Dorée en 1938. Poète et auteur de chansons. A publié une vingtaine de livres dont Un peu plus d'ombre au dos de la falaise (Estérel, 1966 - prix Du Maurier) et Mon refuge est un volcan (l'Hexagone, 1977 - prix du Gouverneur général).

\section{Claudette Laprise}

Née à Sherbrooke. Enseigne au Collège de Sherbrooke.

\section{Gilles Leclerc}

Né à Saint-Rosaire d'Arthabaska en 1928. Poète, éditeur, journaliste et essayiste. A publié, entre autres, La chair abolie (Ed. de l'Aube, 1957) et Journal d'un inquisiteur (id., 1960; réédité en 1974). Quasi-fondateur, avec Maurice Beaulieu, en 1963, de l'Office de la langue française - où il travaille toujours -, à la suite du départ de Jean-Marc Léger.

\section{Gordon Lefebure}

Né à Cornwall (Ontario) en 1944. A étudié la traduction à l'Université McGill. Initiateur, avec Laurent-Michel Vacher et André Roy, du magazine Spirale. Enseigne à l'Université du Québec à Montréal.

\section{Liliane Le Grand}

Née au Manitoba en 1928. En Ontario pendant onze ans, ã Montréal depuis 1960. A étudié la traduction à l'Université de Montréal. Actuellement traductrice à la pige. 


\section{André Marquis}

Né à Granby en 1960. Poète et critique. A publié Si l'herbe pour toujours (Soudeyns-Donzé, 1984) et Le jeu sans fin (XYZ, 1985). Chargé de cours à l'Université de Sherbrooke et au Séminaire de Sherbrooke.

\section{Danielle Mazé}

Née à Mostaganem (Algérie). Prépare un doctorat en littérature canadienne comparée à $l^{i}$ Université de Sherbrooke. Enseigne les langues au Lycée de Nouméa (NouvelleCalédonie).

\section{Robert Mélançon}

Né à Montréal en 1947. Poète et critique. A publié Peinture aveugle (VLB, 1979 -prix du Gouverneur général). Une traduction de poèmes d'Earle Birney est sous presse chez le même éditeur. Travaille, en collaboration avec Charlotte Mélançon, à une traduction de The Second Scroll d'A.M. Klein. Enseigne à l'Université de Montréal.

\section{Françoise Nadeau}

Née à Saint-Pierre de Broughton en 1955. Son mémoire de maîtrise en littérature canadienne comparée à l'Université de Sherbrooke, qui consistait en la traduction d'un roman canadien, vient de paraître: Danse à contre-jour de Joan Barfoot (Ed. Québec/Amérique).

\section{Robert Paquin}

Né à Montréal en 1946. Nationalité: canadienne. Numéro d'assurance sociale: 219040 284. Taille: $1,78 \mathrm{~m}$. Masse: $63 \mathrm{~kg}$. Président de l'Association des traducteurs littéraires du Canada.

\section{Suzanne Paradis}

Née à Québec en 1936. Poète, romancière, critique et essayiste. A publié une vingtaine de livres dont Pour les enfants des morts (Garneau, 1964 - prix France-Canada) et Un goût de sel (Leméac, 1984 - prix du Gouverneur général).

\section{Bernard Pozier}

Né à Trois-Rivières en 1955. Poète et critique. A publié une dizaine de livres dont Caroline Romance (Ed. Arcade, 1983) et Bacilles de tendresse (Ecrits des Forges, 1985). Directeur littéraire aux Écrits des Forges. Enseigne au Collège Joliette-de Lanaudière.

\section{Claudine Richetin}

Née à Sury-en-Vaux (Cher, France) en 1947. Deux passions: le théâtre et la traduction. Enseigne l'anglais dans une école secondaire à Riom (France).

\section{Carole Rondeau}

Née à Saint-Hyacinthe en 1948. Séjour prolongé aux États-Unis, suivi d'études universitaires «sur le tard» à l'Université de Sherbrooke. Pigiste en toutes sortes de domaines puis, finalement, entrepreneure en traduction... et en «Rédaction etc.» (enr.). 


\section{André Roy}

Né à Montréal en 1944. Poète et critique de cinéma. A publié une vingtaine de livres dont Les passions du samedi (Ed. Les Herbes rouges, 1979) et Action writing (id., 1985 - prix du Gouverneur général). A participé avec 3 poètes québécois et 4 poètes canadiens-anglais au numéro 29/30 (intitulé La traduction à l'épreuve/Experiment in translation) de la revue Ellipse. Chargé de cours à l'Université Concordia.

\section{Mario Savoie}

Né dans la région de Joliette en 1960. Étudie actuellement à l'Université de Montréal en sciences de la santé. Infirmier à l'Hôpital Saint-Luc.

\section{Harold Smyth}

Né à Saint-Basile (Nouveau-Brunswick) en 1939. Maître de chapelle à la cathédrale Saint-Germain à Rimouski. Enseigne l'anglais à l'École polyvalente Paul-Hubert à Rimouski.

\section{Robert Yergeau}

Né à Cowansville en 1956. Poète et critique. A publié, entre autres, L'oralité de l'émeute (Naaman, 1981 - prix Gaston-Gouin) et L'usage du réel suivi de Exercices de tir (Ed. du Noroît, 1986). Enseigne au Collège universitaire de Hearst (Ontario). 